DE

M E D I C I N A

T R O P I C A L

$\mathrm{DE}$

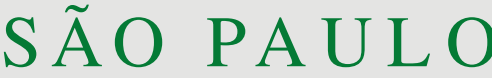

JOURNAL OF THE SÃO PAULO INSTITUTE OF TROPICAL MEDICINE

${ }^{1}$ Ministry of Public Health, National Institute of Health, Department of Medical Sciences, Nonthaburi, Thailand

${ }^{2}$ Ministry of Public Health, Department of Disease Control, Bureau of Tuberculosis, Bangkok, Thailand

Correspondence to: Benjawan Phetsuksiri Ministry of Public Health, National Institute of Health, Department of Medical Sciences, 88/7 Tiwanon Rd, 11000, Muang, Nonthaburi, Thailand

Tel: +66 025801567

Fax: +66 029659700

E-mail: benjapsk@ health.moph.go.th

Received: 10 May 2018

Accepted: 30 August 2018

\section{QuantiFERON-TB Gold In-Tube test in active tuberculosis patients and healthy adults}

Benjawan Phetsuksiri', Sopa Srisungngam ${ }^{1}$, Janisara Rudeeaneksin', Supranee Boonchu ${ }^{1}$, Wiphat Klayut ${ }^{1}$, Ronnayuth Norrarat ${ }^{2}$, Somchai Sangkitporn ${ }^{1}$, Yuthichai Kasetjaroen ${ }^{2}$

\section{ABSTRACT}

Interferon-gamma (IFN- $\gamma$ ) release assays have improved latent tuberculosis (TB) detection and have been considered promising for the diagnosis of TB disease. However, diagnosis efficacy data is limited in high burden countries. The aim of this study was to determine the diagnostic potential of the QuantiFERON-TB Gold In-Tube (QFT-GIT) test for the diagnosis of active TB in an endemic setting for TB. A cross-sectional study was conducted in a group of 102 Thai patients with clinical symptoms and chest x-ray findings suggesting of active pulmonary TB and a group of 112 healthy adults. Testing was carried out using sputum microscopy, mycobacterial culture and QFT-GIT test. Of these patients, QFT-GIT was positive in $73(71.57 \%)$, negative in $27(26.47 \%)$, and undetermined in $2(1.96 \%)$ cases. Among healthy controls, QFT-GIT was positive in 18 (16.07\%), negative in $93(83.04 \%)$, and undetermined in $1(0.89 \%)$ person. Based on TB culture results, the sensitivity of QFTGIT for diagnosing active TB was $84.21 \%$ (95\% confidence interval (CI); 72.13-92.52). The positive and negative predictive values were $65.75 \%$ (95\% CI; 59.26-71.70) and 66.67\% (95\% CI; 49.94-80.04), respectively. The median IFN- $\gamma$ level in culture-confirmed TB patients was 3.91 compared to $0.03 \mathrm{IU} / \mathrm{mL}$ of the healthy group. QFT-GIT appears to be a useful indirect test for TB diagnosis in Thailand and its use is recommended in association with clinical and radiological assessments for identifying active or latent TB.

KEYWORDS: Active tuberculosis. Latent tuberculosis. Diagnosis. QuantiFERON. Interferon-gamma release assay. IGRA

\section{INTRODUCTION}

Tuberculosis (TB) remains a major global health threat. Despite significant efforts to control the disease, mortality and incidence rates remain extremely high ${ }^{1}$. An essential factor to control the spread of TB is the ability to diagnose the disease at the early stages. Smear microscopy is generally available but it has poor sensitivity. At least, 5,000 bacilli per $\mathrm{mL}$ of sputum are required for a smear-positive result. In addition, a positive finding is not specific only to Mycobacterium tuberculosis. On the other hand, mycobacterial culture, the gold standard, usually takes several weeks until final results. It also requires specific laboratory equipment, technicians with additional skills and appropriate bio-safety conditions ${ }^{2}$. Therefore, sensitive tools complementing conventional tests are needed to guide the initiation of therapy when the diagnosis of TB disease remains doubtful.

Interferon-gamma release assays (IGRAs) are immunodiagnostics tools in which interferon-gamma (IFN- $\gamma$ ) released by T-cells in response to Mycobacterium 
tuberculosis-specific antigen is measured ${ }^{3}$. During the past decade, two commercial in vitro IGRAs, including the QuantiFERON-TB Gold In-Tube (QFT-GIT) test (Cellestis, Ltd., Victoria, Australia) and T-SPOT.TB (Oxford Immunotec, UK), have been introduced for the early detection of M. tuberculosis infection. The QFT-GIT test detects IFN- $\gamma$ production after the stimulation of whole blood samples with specific $M$. tuberculosis antigens, Early Secretory Antigenic Target-6 (ESAT-6), Culture Filtrate Protein-10 (CFP-10) and TB7.7 (Rv2654). These antigens are absent from all Bacille Calmette-Guérin (BCG) strains, as well as most common non-tuberculous mycobacteria. The QFT-GIT has been reported to have higher sensitivity, specificity and be more attractive than the tuberculin skin test (TST) which may result in interpretation errors, including the need of two clinic visits. However, the QFT-GIT performance in detecting active or latent tuberculosis infection (LTBI) can vary depending on the tested populations and regions ${ }^{4}$.

The QFT-GIT test has been largely employed to evaluate the diagnosis of $M$. tuberculosis infection in developed countries ${ }^{4}$. In recent years, the studies of QFT-GIT performance have become more documented in non-developed countries such as Iran, India, Zambia, Brazil, etc. ${ }^{5,6}$. Currently available data suggest that the QFT-GIT test is less influenced by prior BCG vaccination and environmental mycobacteria ${ }^{7}$. Therefore, the QFT-GIT test has been increasingly used in locations where the coverage of BCG vaccination is high. Numerous studies have assessed the utility of this test in diagnosing latent TB infection (LTBI) in various clinical settings ${ }^{8}$. Regarding active TB, the use of IGRAs has been unclear particularly in high prevalence settings. Although the limitations of QFT-GIT in diagnosing TB disease have been recognized, it continues to be recommended by some investigators and used to aid in the diagnosis of tuberculosis ${ }^{9,10}$. Thailand is a TB-endemic country with an estimated prevalence of 172 per 100,000 inhabitants ${ }^{1}$. National guidelines have not yet been established using IGRA for LTBI screening or the diagnosis of TB, while the TST is the test of choice for identifying TB infection. Currently, the QFT-GIT test is available and has been used for the diagnosis of LTBI in Thailand. Nevertheless, it has been used in clinical practice for the rapid confirmation of TB disease or for ruling out active TB in many suspicious cases, especially when there are insufficient or non-sputum samples. Collecting blood samples may not frequently encounter difficulties compared with other specimens collection and QFT-GIT can provide rapid results, within two days. Despite the high cost, QFT-GIT may be a useful additional test for TB diagnosis especially when reference test results are not available. However, data on the utility and the efficacy of QFT-GIT for diagnosing active TB are limited in this setting. Here, we assessed the diagnostic potential of the QFT-GIT assay for detecting TB infection among the active TB patients in Thailand, with healthy adults as the controls.

\section{MATERIALS AND METHODS}

\section{Study subjects}

This study was reviewed and approved by the Ethics Committee of the Ministry of Public Health, Thailand and was conducted from September 2013 through October 2015. The participants were clinically diagnosed with pulmonary tuberculosis (PTB) and there was a group of healthy subjects. Informed consent was obtained from all participants before the enrollment in this study. Data concerning primary demographic characteristics and sputum smear results were collected.

Eligible patients included presumptive active TB cases (PTB) with sputum smear positive or negative and aged over 15 years. The presence of active TB was defined as the presence of abnormal images suggestive of TB in the chest $\mathrm{x}$-ray or clinical manifestations such as a persistent cough for more than two weeks, night sweats, prolonged fever, weight loss or hemoptysis, with or without a history of TB exposure. As the disease could not be confirmed by bacteriological culture at the time of recruitment, the physicians determined whether the patients suffered from TB based on clinical, microbiological and radiological findings. For patients considered as suspected cases of PTB, if the sputum culture was positive they were diagnosed as certain TB patients. Subjects were recruited to this study consecutively. To avoid misinterpretation due to an immune response modulation, any patient who had previously received anti-tuberculous treatment, steroids, anti-TNF compounds or any other medication that might reduce the cellular immunity was excluded from the study. Patients with self-reported disease or presenting symptoms of diseases that compromise the immune system such as HIV, diabetes or cancer were not eligible. Clinical examination and screening for other underlying diseases were performed following routine guidelines.

The healthy subject group included adults in good physical conditions aged over 15 years, with no apparent TB symptoms, abnormal chest X-ray findings, history of $\mathrm{TB}$ or exposure to the disease. Their contact with physicians consisted primarily of annual medical check-ups.

\section{Specimen collection and mycobacteriology}

As part of a routine procedure for TB diagnosis, three 
sputum samples, the first sputum, second early morning and the third sputum were collected from the patients, with all subjects taken for radiological examination. The collected sputum specimens were stained for acid-fast bacilli (AFB) microscopy by Ziehl-Neelsen method, and decontaminated samples were cultured in Lowenstein-Jensen (LJ), a conventional medium for the isolation and identification of M. tuberculosis ${ }^{11}$. Blood samples were drawn from all recruited study subjects for QFT-GIT at the time of standard microscopic examination, after which the QFT-GIT assay was carried out.

\section{QFT-GIT assay}

The whole-blood assay of QFT-GIT was performed according to the manufacturer's instructions (Cellestis, Ltd., Victoria, Australia). Briefly, a total of $3 \mathrm{~mL}$ of peripheral venous blood was taken from each subject and immediately transferred to 3 QFT-GIT tubes of $1 \mathrm{~mL}$ each (Nil controlno antigen for negative control, phytohaemagglutinin or mitogen for positive control and TB-specific antigens). Then, the tubes were homogenized by inversion for ten times and were subsequently incubated for $16-24 \mathrm{~h}$ (targeting 16-18 $\mathrm{h}$ incubation time in our study) at $37^{\circ} \mathrm{C}$. Plasma samples were recovered after centrifugation and stored at $4{ }^{\circ} \mathrm{C}$ until assayed. QFT-GIT enzyme-linked immunosorbent assay (ELISA) was carried out to measure IFN- $\gamma$ within 2 weeks after blood collection. Raw optical densities were interpreted using specific software supplied by the manufacturer (QuantiFERON-TB Gold analysis software version 2.50.4). The results obtained by the Nil control were subtracted from the mitogen control and the antigen-stimulated samples. The cut-off point for the diagnosis was set according to the manufacturer's instructions. In brief, if the IFN- $\gamma$ secretion in response to TB antigens after subtracting Nil control IFN- $\gamma$ was $0.35 \mathrm{IU} / \mathrm{mL}$, it was considered positive. If the value was less than $0.35 \mathrm{IU} / \mathrm{mL}$, it was considered negative. The result of the test was considered undetermined if an antigen-stimulated sample was negative and the value of the positive control was less than $0.5 \mathrm{IU} / \mathrm{mL}$. Subjects with IFN- $\gamma$ secretion over $8.0 \mathrm{IU} / \mathrm{mL}$ in the Nil control samples were also considered undetermined for QFT-GIT.

\section{Statistical analysis}

Numbers, frequencies, percentages and medians were used to present data. The association between QFT-GIT results of the two groups was determined by the Chi-square test or the Fisher's exact test using SAS ${ }^{\oplus}$ University Edition. A $p$-value less than 0.05 was considered a statistically significant difference. The diagnostic performance of the QFT-GIT test was assessed using sensitivity and positive/ negative predictive values. Sensitivity was calculated without undetermined results.

\section{RESULTS}

\section{Demographic and microbiological characteristics of study subjects}

A total of 214 subjects were enrolled in the study. Of these participants, 112 were healthy adults and 102 were new clinically diagnosed PTB patients. The demographic profile of TB patients and healthy controls is shown in Table 1. Patients and healthy controls had a median age of 37 (range, 15-68 years) and 42 (range, 15-76), respectively, and they were not age-matched. Sixty-nine of $102(67.65 \%)$ patients and 76 of $112(67.86 \%)$ healthy adults were men. Sputum smear microscopy was positive in 53 of 102 (51.96\%) patients, while smear was negative in 49 of 102 (48.04\%). Culture for M. tuberculosis was positive in 59 of 102 , therefore, the culture positive rate in this study was $57.84 \%$. Based on culture confirmation for M. tuberculosis, TB cases were definitively identified in a total of 59 cases, of which 48 and 11 were AFB smear positive and negative, respectively (Table 2).

Table 1 - Demographic and MTB microbiological characteristics of study subjects

\begin{tabular}{|c|c|c|}
\hline \multirow[b]{2}{*}{ Variables } & \multicolumn{2}{|c|}{ Number of subjects } \\
\hline & $\begin{array}{l}\text { PTB patients } \\
\quad(n=102)\end{array}$ & $\begin{array}{l}\text { Healthy adults } \\
\quad(n=112)\end{array}$ \\
\hline \multicolumn{3}{|l|}{ Gender } \\
\hline Female & $33(32.35)$ & $36(32.14)$ \\
\hline Male & 69 (67.65) & $76(67.86)$ \\
\hline Age (min-max), median & (15-68), 37 & $(15-76), 42$ \\
\hline $15-25$ & 19 (18.63) & $20(17.86)$ \\
\hline $26-35$ & $30(29.41)$ & $23(20.54)$ \\
\hline $36-45$ & $21(20.59)$ & $32(28.57)$ \\
\hline $46-60$ & $27(26.47)$ & $29(25.89)$ \\
\hline $61-76$ & $4(3.92)$ & $8(7.14)$ \\
\hline Unknown & $1(0.98)$ & - \\
\hline \multicolumn{3}{|l|}{ Sputum smear status } \\
\hline Smear positive & $53(51.96)$ & - \\
\hline Smear negative & $49(48.04)$ & - \\
\hline \multicolumn{3}{|l|}{ MTB Culture status } \\
\hline Culture positive & $59(57.84)$ & - \\
\hline Culture negative & $43(42.16)$ & - \\
\hline
\end{tabular}


Table 2 - QuantiFERON-TB Gold In-Tube (QFT-GIT) results in PTB patients and healthy subjects

\begin{tabular}{lccccc}
\hline Subjects & Number of subjects & Positive (\%) & Negative (\%) & Indeterminate (\%) & $p$-value \\
\hline Suspected PTB & 102 & $73(71.57)$ & $27(26.47)$ & $2(1.96)$ & $<0.0001$ \\
S+, C+ & 48 & $40(83.33)$ & $6(12.5)$ & $2(4.17)$ & $<0.0001$ \\
S+, C- & 5 & $3(60.0)$ & $2(40.0)$ & - & $0.04^{\mathrm{b}}$ \\
S-, C+ & 11 & $8(72.73)$ & $3(27.27)$ & - & $0.0002^{\mathrm{b}}$ \\
S-, C- & 38 & $22(57.89)$ & $16(42.11)$ & - & $<0.0001$ \\
Healthy adults & 112 & $18(16.07)$ & $93(83.04)$ & $1(0.89)$ & \\
\hline
\end{tabular}

${ }^{a} \mathrm{QFT}$-GIT results in patients clinically diagnosed having PTB compared to those of healthy controls using Chi-square or Fisher's exact $^{b}$ test. PTB, pulmonary tuberculosis; S, acid-fast bacilli smear result; C, culture result. The patient group was stratified based on results of sputum microscopy and mycobacterial culture

\section{Performance of QFT-GIT for TB diagnosis}

Overall, 73 (71.57\%), 27 (26.47\%) and $2(1.96 \%)$ of the cases had positive, negative and undetermined QFT-GIT results, respectively compared with 18 (16.07\%), 93 (83.04\%) and $1(0.89 \%)$ of the controls respectively, with $p$-values $<0.0001$ for positive and negative results. In subgroup analysis, the positive rate of QFT-GIT for the diagnosis of active PTB in the group of smear-positive and culture-positive cases was 40 of $48(83.33 \%)$. The positive rate for QFT-GIT in detecting smear-negative and culture-positive PTB patients was 8 of 11 or $72.73 \%$. In culture-negative patients, positive QFT-GIT was $60 \%$ and $57.89 \%$ in the group of AFB positive and negative, respectively. The number of positive results for each group of PTB patients obtained by the IFN- $\gamma$ assay is shown in Table 2. The difference(s) in the percentages of QFT-GIT results for the IFN- $\gamma$ test in each group of patients compared with those of the controls were significant ( $p$-values $<0.05$ ). A undetermined or uninterpretable result was found among the group of active PTB patients in two cases due to the low immune response to mitogen antigen in a positive control tube. Of the 112 healthy subjects, 18 (16.07\%) had an IFN- $\gamma$ level over $0.35 \mathrm{IU} / \mathrm{mL}$, indicating probable M. tuberculosis infection or LTBI. One healthy adult had an IFN- $\gamma$ level over $0.35 \mathrm{IU} / \mathrm{mL}$, but less than $25 \%$ of the Nil value. The interpretation of this result was QFT-GIT negative. A undetermined result caused by the low immune response was also found in only one subject among the healthy controls $(0.89 \%)$. Retesting of these samples has not been performed and all three undetermined results were excluded from further analysis. There was a significant difference of positive QFT-GIT results between the TB patient group and the asymptomatic or non-TB, healthy group, $p$-value $<0.05$. The sensitivity and predictive values for the diagnosis of active TB were calculated by excluding two culture positive cases presenting undetermined results for QFT-GIT. Among the remaining 57 culture-positive patients, 48 had a QFT-GIT positive result. Therefore, the sensitivity of QFT-GIT in culture-confirmed TB cases was 84.21\% (95\% CI; 72.13-92.52), whereas the sensitivity in the patient group with AFB smear-positive, regardless of the culture result, was $84.31 \%$ (95\% CI; 71.41-92.98) (Table 3). Further analysis based on clinical symptoms, the smear microscopy result and radiological evaluation indicated that the sensitivity of QFT-GIT for the detection of active TB disease was 73.0\% (95\% CI 63.20-81.39). The positive predictive values (PPV) and the negative predictive value (NPV) are detailed in Table 3.

\section{Level of IFN- $\gamma$ between patient and control groups}

The levels of IFN- $\gamma$ in response to TB specific antigens in PTB patients and healthy adults are shown in Figure 1. There was an overlapping of IFN- $\gamma$ levels between the groups of active TB disease and asymptomatic infected or LTBI. Four of LTBI showed very high levels of IFN- $\gamma$, over $10.0 \mathrm{IU} / \mathrm{mL}$, thus the level of IFN- $\gamma$ cannot be used alone to distinguish active from latent TB. Apparently, the analysis of IFN- $\gamma$ levels in PTB patients revealed that most active TB cases $(83.56 \%)$ in this study were positive to QFT-GIT with IFN- $\gamma$ levels over $1.0 \mathrm{IU} / \mathrm{mL}$. In culture confirmed PTB, the median IFN- $\gamma$ level was 3.91 compared to $0.03 \mathrm{IU} / \mathrm{mL}$ of the healthy group. Among the healthy group, the median IFN- $\gamma$ levels in LTBI and in non-LTBI were $1.44 \mathrm{IU} / \mathrm{mL}$, and $0.02 \mathrm{IU} / \mathrm{mL}$, respectively. The median IFN- $\gamma$ levels in patients with smear positive and culture positive $(\mathrm{S}+\mathrm{C}+)$, smear positive and culture negative ( $\mathrm{S}+\mathrm{C}-$ ), smear negative and culture positive (S-C+), including smear negative and culture negative (S-C-), were $3.24,0.52,4.67,0.71 \mathrm{IU} / \mathrm{mL}$, respectively.

\section{DISCUSSION}

The measurement of IFN- $\gamma$ in antigen-stimulated blood samples by QFT-GIT has been proposed as an in vitro 
Table 3 - Diagnostic performance of the QuantiFERON-TB Gold In-Tube (QFT-GIT) test in active tuberculosis patients compared with microbiological results and clinical TB diagnosis

\begin{tabular}{|c|c|c|c|c|c|c|}
\hline \multirow{2}{*}{\multicolumn{2}{|c|}{ Number of subjects $\left(102^{a}\right)$}} & \multicolumn{2}{|c|}{ MTB culture } & \multirow{2}{*}{$\begin{array}{l}\text { \%sensitivity } \\
(95 \% \mathrm{Cl})\end{array}$} & \multirow{2}{*}{$\begin{array}{c}\% \text { PPD } \\
(95 \% \mathrm{Cl})\end{array}$} & \multirow{2}{*}{$\begin{array}{c}\text { \%NPD } \\
(95 \% \mathrm{Cl})\end{array}$} \\
\hline & & Positive & Negative & & & \\
\hline \multirow{2}{*}{ QFT-GIT } & Positive & 48 & 25 & \multirow{2}{*}{$\begin{array}{c}84.21 \\
(72.13-92.52)\end{array}$} & \multirow{2}{*}{$\begin{array}{c}65.75 \\
(59.26-71.70)\end{array}$} & \multirow{2}{*}{$\begin{array}{c}66.67 \\
(49.94-80.04)\end{array}$} \\
\hline & Negative & 9 & 18 & & & \\
\hline \multirow{2}{*}{\multicolumn{2}{|c|}{ Number of subjects $\left(102^{b}\right)$}} & \multicolumn{2}{|c|}{ AFB smear } & & & \\
\hline & & Positive & Negative & & & \\
\hline \multirow{2}{*}{ QFT-GIT } & Positive & 43 & 30 & \multirow{2}{*}{$\begin{array}{c}84.31 \\
(71.41-92.98) \\
\end{array}$} & \multirow{2}{*}{$\begin{array}{c}58.90 \\
(52.69-64.85)\end{array}$} & \multirow{2}{*}{$\begin{array}{c}70.37 \\
(53.44-83.09)\end{array}$} \\
\hline & Negative & 8 & 19 & & & \\
\hline \multirow{2}{*}{\multicolumn{2}{|c|}{ Number of subjects $\left(214^{c}\right)$}} & \multicolumn{2}{|c|}{ Clinical Dx TB } & & & \\
\hline & & Suspected & Healthy & & & \\
\hline \multirow{2}{*}{ QFT-GIT } & Positive & 73 & 18 & \multirow{2}{*}{$\begin{array}{c}73.00 \\
(63.20-81.39)\end{array}$} & \multirow{2}{*}{$\begin{array}{c}80.22 \\
(72.33-86.29)\end{array}$} & \multirow{2}{*}{$\begin{array}{c}77.50 \\
(71.18-82.77)\end{array}$} \\
\hline & Negative & 27 & 93 & & & \\
\hline
\end{tabular}

a2 from MTB culture positive were indeterminate by QFT-GIT; b2 from AFB smear positive were indeterminate by QFT-GIT; ${ }^{\mathrm{c}} 2$ from suspected tuberculosis cases and one from healthy adults were indeterminate by QFT-GIT. MTB, Mycobacterium tuberculosis; CI, confidential interval; PPD, positive predictive value; NPV, negative predictive value; AFB, acid-fast bacilli; Dx, diagnosis

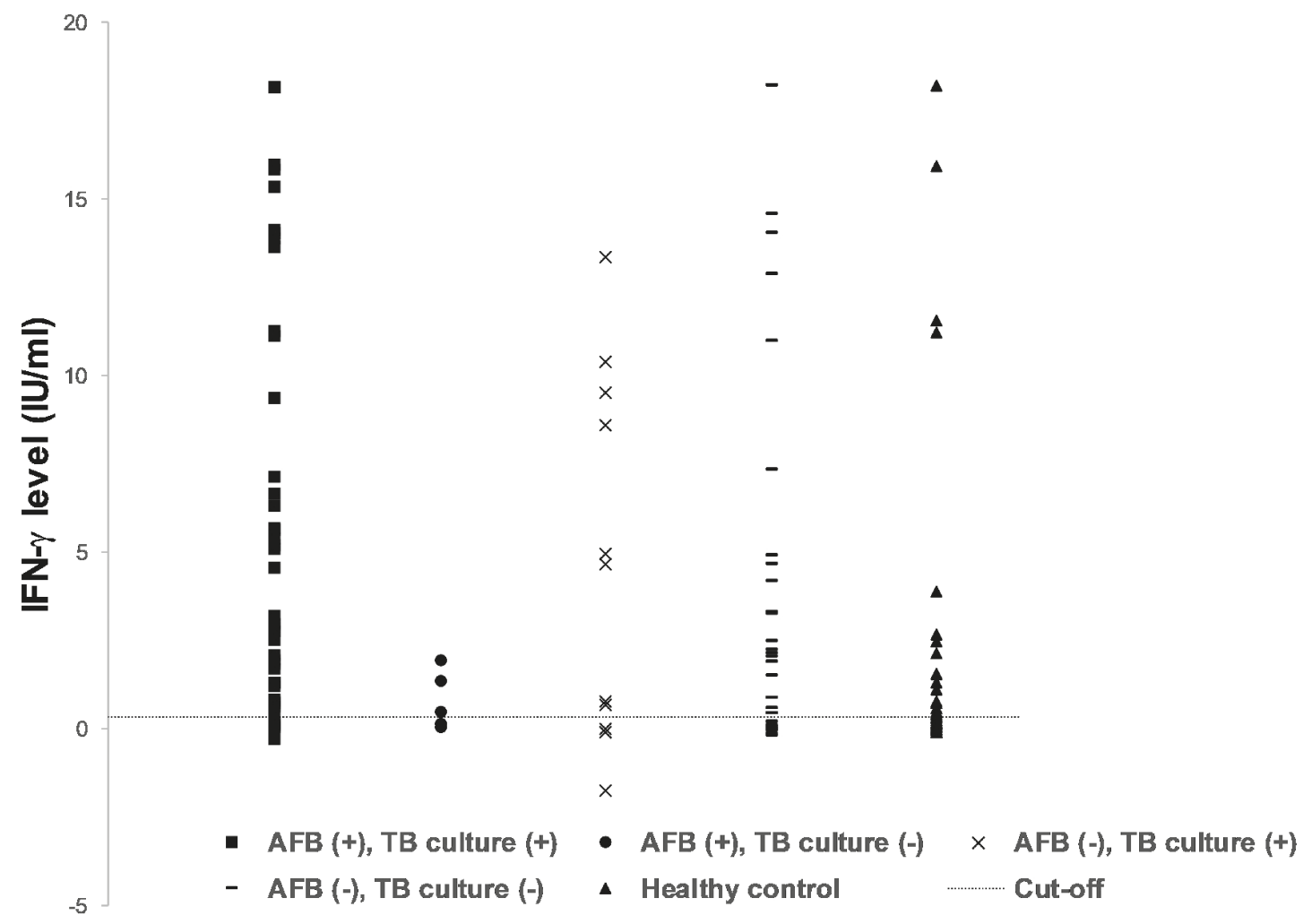

Figure 1 - Quantitative responses of the QuantiFERON-TB Gold In-Tube test in pulmonary tuberculosis patients in relation to acid-fast bacilli smear and culture results compared with the responses of healthy adults. Individual QFT-GIT results are plotted according to their diagnosis. The cut-off value for positive QFT-GIT is represented by the dotted line $(0.35 \mathrm{IU} / \mathrm{mL})$

alternative method for latent TB diagnosis and has the potential to provide rapid and reliable results to diagnose active $\mathrm{TB}^{12-14}$. There are several published reports on the performance of the QFT-GIT test for TB diagnosis used in non-endemic countries ${ }^{15}$ but few studies have been carried out in TB or TB-HIV endemic settings ${ }^{16}$. This study reported the use of the QFT-GIT assay in Thailand, where the burden of TB is high and BCG vaccination is commonly employed. In this study, the positive rate of QFT-GIT was $84.21 \%$ in culture-positive cases and $84.31 \%$ in AFB smear- 
positive group, which was in the range described in some previous reports ${ }^{17-20}$. Previously published studies have also demonstrated the very high specificity of QFT-GIT, giving no reason to doubt the validity of QFT-GIT results for detecting MTB infection ${ }^{21,22}$. The high background of LTBI may result in poor specificity for active tuberculosis diagnosis in high-burden countries. In this study, we reported the percentage of LTBI to be $16.07 \%$. It has been recommended that QFT-GIT could be used to rule out the suspicion of active TB disease among clinically suspected subjects $^{23}$. In this condition, those suspected of having TB would show low levels of IFN- $\gamma(<0.35 \mathrm{IU} / \mathrm{mL})$. It was proposed that QFT-GIT could be used as a confirmatory test for active TB disease at the moment of the clinical diagnosis, before culture results are available ${ }^{17}$. QFT-GIT is less influenced by prior BCG vaccination and environmental mycobacteria ${ }^{3,7}$. Therefore, high rates of $B C G$ vaccination should not be a concern of use in this setting and elsewhere. QFT-GIT has considerably high sensitivity, comparable with or superior to that of TST. In addition, the sensitivity of QFT-GIT has been reported to be higher for detecting active TB disease in low endemic countries ${ }^{24-26}$. The positive rate of QFT-GIT in detecting active TB in Thailand reported in this study appeared to be similar to those of other countries. The slight differences in sensitivity found by our study and others may be explained by the difference in the number of analyzed patients and their individual characteristics. The false-negative results of QFT-GIT in active TB have been described previously ${ }^{27-29}$. Some hypotheses have been raised to explain the negative results in active TB patients. Immunocompromised patients with active TB at the beginning of the treatment were also reported and they were more likely to have negative QFT-GIT results. In immunocompetent patients with active TB but negative QFT-GIT, the explanation for this finding involves the release of anti-inflammatory cytokines by peripheral blood mononuclear cells and a temporary depression of the T-cell response. HIV is one of the major influencing factors for QFT-GIT diagnostic results ${ }^{30}$. Diabetes mellitus is another factor affecting the expression of Th1-related cytokines ${ }^{31}$. In addition, it was reported that QFT-GIT response could be diminished in cases of advanced $\mathrm{TB}^{32}$. Therefore, negative QFT-GIT test results should not be used alone to exclude active TB. For LTBI, as QFT-GIT is recommended for LTBI detection, a prevalence of LTBI should be further investigated and considered for appropriate management in endemic countries.

The limitations of this study included the small sample size, the lack of sociodemographic data, no molecular results to rapidly confirm the clinical diagnosis of tuberculosis and the absence of clinical information regarding the routine screening of other underlying diseases, as well as of the HIV status of study patients. We noted that the culture sensitivity in the present study was as low as $57.8 \%$. The sensitivity of mycobacterial cultures can vary from $20-100 \%^{33}$ and in a similar study, the sensitivity of MTB sputum culture was $60 \%{ }^{34}$. Therefore, the culture sensitivity in our study was not out of this range. Another point that worths discussion is the role of culture as the gold standard, as it may sometimes result in false negative results due to poor sputum sample collection or paucibacillary sputum samples. Although the study had some limitations, the results demonstrated the usefulness of QFT-GIT when used in TB high-prevalence settings for diagnosis of M. tuberculosis infections. It is more useful in the case of smear-negative PTB patients and can help to rule out active pulmonary TB suspicions. Nevertheless, the QFT-GIT assay can result in false negative or undetermined results in active TB with low T-lymphocytes response. Therefore, QFT-GIT should not be used alone or as the final test for the identification of active TB patients. Culture results are still required for conclusive TB diagnosis. We have also shown that QFT-GIT could not discriminate active TB disease from latent TB infection alone. Undetermined results were found in a small number of patients in this study. It was reported that QFT-GIT undetermined results increase with age and are associated with the severity of other underlying diseases in patients with active TB disease. Because of the small number of elderly patients, the study had insufficient data to address this issue. For further studies, the testing in environmental mycobacteriosis and in HIV-infected patients in Thailand is proposed.

\section{CONCLUSIONS}

In general, there is concern about the usefulness of QFT-GIT for active TB diagnosis in TB-endemic countries. Based on our results, we conclude that QFT-GIT is useful as a complementary test for diagnosing active TB cases. It appeared to be useful for detecting latent tuberculosis in a high prevalence setting country as Thailand. IFN- $\gamma$ levels could not distinguish between active and latent TB infection. Therefore, QFT-GIT cannot be used separately without considering clinical and radiological data for identifying active or latent TB. There is still much to learn on the QFTIT assay and its performance in different clinical settings, meaning that the subject deserves further study.

\section{ACKNOWLEDGMENTS}

The authors wish to thank all the study subjects for their willingness to participate in this study. This 
work was supported by contributions from a team that included clinical nurses and laboratory technicians. Their contributions are acknowledged.

This research was funded by the National Institute of Health, Department of Medical Sciences, Ministry of Public Health, Thailand.

\section{REFERENCES}

1. World Health Organization. Global tuberculosis report 2017. Geneva: WHO; 2017. [cited 2018 Aug 30] Available from: http://www.who.int/tb/publications/global_report/en/

2. World Health Organization. Implementing tuberculosis diagnostics: a policy framework. Geneva: WHO; 2015. [cited 2018 Aug 30] Available from: http://www.who.int/tb/ publications/implementing_TB_diagnostics/en/

3. Lalvani A. Diagnosing tuberculosis infection in the $21^{\text {st }}$ century: new tools to tackle an old enemy. Chest. 2007;131:1898-906.

4. Lu P, Chen X, Zhu LM, Yang HT. Interferon-gamma release assays for the diagnosis of tuberculosis: a systematic review and meta-analysis. Lung. 2016;194:447-58.

5. Metcalfe JZ, Everett CK, Steingart KR, Cattamanchi A, Huang $\mathrm{L}$, Hopewell PC, et al. Interferon- $\gamma$ release assays for active pulmonary tuberculosis diagnosis in adults in low- and middleincome countries: systematic review and meta-analysis. J Infect Dis. 2011;204 Suppl 4:S1120-9.

6. Ferreira TF, Matsuoka PF, Santos AM, Caldas AJ. Diagnosis of latent Mycobacterium tuberculosis infection: tuberculin test versus interferon-gamma release. Rev Soc Bras Med Trop. 2015;48:724-30.

7. Starke JR, Committee On Infectious Diseases. Interferon- $\gamma$ release assays for diagnosis of tuberculosis infection and disease in children. Pediatrics. 2014;134:e1763-73.

8. Diel R, Goletti D, Ferrara G, Bothamley G, Cirillo D, Kampmann $\mathrm{B}$, et al. Interferon- $\gamma$ release assays for the diagnosis of latent Mycobacterium tuberculosis infection: a systematic review and meta-analysis. Eur Respir J. 2011;37:88-99.

9. Dai Y, Feng Y, Xu R, Xu W, Lu W, Wang J. Evaluation of interferon-gamma release assays for the diagnosis of tuberculosis: an updated meta-analysis. Eur J Clin Microbiol Infect Dis. 2012;31:3127-37.

10. Anwar A, Hamdan AJ, Salim B, Yosra A, Hani M, Abdullah AH. Diagnostic utility of QuantiFERON-TB Gold (QFT-G) in active pulmonary tuberculosis. J Glob Infect Dis. 2015;7:108-12.

11. Kent PT, Kubica GP. Public health mycobacteriology: a guide for the level III laboratory. Atlanta: Centers for Disease Control; 1985.

12. Adewole OO, Erhabor GE, Sogaolu MO, Onipede AO, Owiafe PK, Awopeju FO, et al. Diagnostic utility of QuantiFERONTB Gold In-Tube in active pulmonary tuberculosis in Nigeria. West Afr J Med. 2013;32:180-5.
13. Sauzullo I, Mengoni F, Ermocida A, Massetti AP, D’Agostino C, Russo G, et al. Interferon- $\gamma$ release assay in HIV-infected patients with active tuberculosis: impact of antituberculous drugs on host immune response. New Microbiol. 2014;37:15361.

14. Laurenti P, Raponi M, de Waure C, Marino M, Ricciardi W, Damiani G. Performance of interferon $-\gamma$ release assays in the diagnosis of confirmed active tuberculosis in immunocompetent children: a new systematic review and meta-analysis. BMC Infect Dis. 2016;16:131.

15. Lavender TW, Barrett A, Magee J, Ong EL. Interferon- $\gamma$ release assays in the diagnosis of active tuberculosis disease in a lowincident setting: a 5-year review of data. Clin Microbiol Infect. 2013;19:1078-81.

16. Dheda K, van Zyl Smit R, Badri M, Pai M. T-cell interferongamma release assays for the rapid immunodiagnosis of tuberculosis: clinical utility in high-burden vs low-burden settings. Curr Opin Pulm Med; 2009;15:188-200.

17. Rutherford M, Alisjahbana B, Maharani W, Sampurno H, van Crevel R, Hill PC. Sensitivity of the Quantiferon-Gold In-Tube assay in sputum smear positive TB cases in Indonesia. PLoS One. 2010;5:e12020.

18. James PM, Ganaie FA, Kadahalli RL. The performance of QuantiFERON-TB Gold In-Tube (QFT-IT) test compared to tuberculin skin test (TST) in detecting latent tuberculosis infection (LTBI) in the presence of HIV coinfection in a high TB-burden area with BCG-vaccinated population. J Int Assoc Provid AIDS Care. 2014;13:47-55.

19. Chen J, Zhang R, Wang J, Liu L, Zheng Y, Shen Y, et al. Interferongamma release assays for the diagnosis of active tuberculosis in HIV-infected patients: a systematic review and meta-analysis. PLoS One. 2011;6:e26827.

20. Uzunhan O, Törün SH, Somer A, Salman N, Köksalan K. Comparison of tuberculin skin test and QuantiFERON ${ }^{\circledR}$-TB Gold In-Tube for the diagnosis of childhood tuberculosis. Pediatr Int. 2015;57:893-6.

21. Adetifa IM, Lugos MD, Hammond A, Jeffries D, Donkor S, Adegbola RA, et al. Comparison of two interferon gamma release assays in the diagnosis of Mycobacterium tuberculosis infection and disease in The Gambia. BMC Infect Dis. 2007;7:122.

22. Menzies D, Pai M, Comstock G. Meta-analysis new tools for diagnosis of latent tuberculosis infection: areas of uncertainty and recommendations for research. Ann Intern Med. 2007; 146:340-54.

23. Danel C, Kabran M, Inwoley A, Badje A, Herrmann JL, Moh $\mathrm{R}$, et al. QuantiFERON-TB Gold: performance for ruling out active tuberculosis in HIV-infected adults with high CD4 count in Côte d'Ivoire, West Africa. PLoS One. 2014;9:e107245.

24. Bartu V, Havelkova M, Kopecka E. QuantiFERON-TB Gold in the diagnosis of active tuberculosis. J Int Med Res. 2008;36:434-7. 
25. Harada N, Higuchi K, Yoshiyama T, Kawabe Y, Fujita A, Sasaki $\mathrm{Y}$, et al. Comparison of the sensitivity and specificity of two whole blood interferon-gamma assays for M. tuberculosis infection. J Infect. 2008;56:348-53.

26. Hermansen T, Lillebaek T, Hansen AB, Andersen PH, Ravn P. QuantiFERON-TB Gold In-Tube test performance in Denmark. Tuberculosis (Edinb). 2014;94:616-21.

27. Richeldi L. An update on the diagnosis of tuberculosis infection. Am J Respir Crit Care Med. 2006;174:736-42.

28. Dewan PK, Grinsdale J, Kawamura LM. Low sensitivity of a whole-blood interferon-gamma release assay for detection of active tuberculosis. Clin Infect Dis. 2007;44:69-73.

29. Bao L, Li T, Diao N, Shen Y, Shao L, Zhang Y, et al. Fluctuating behavior and influential factors in the performance of the QuantiFERON-TB Gold In-Tube assay in the diagnosis of tuberculosis. PLoS One. 2015;10:e103763.

30. Huo ZY, Peng L. Accuracy of the interferon- $\gamma$ release assay for the diagnosis of active tuberculosis among HIV-seropositive individuals: a systematic review and meta-analysis. BMC Infect Dis. 2016;16:350.
31. Tsukaguchi K, Okamura H, Matsuzawa K, Tamura M, Miyazaki $\mathrm{R}$, Tamaki S, et al. Longitudinal assessment of IFNgamma production in patients with pulmonary tuberculosis complicated with diabetes mellitus. Kekkaku. 2002;77:409-13.

32. Jeon YL, Nam YS, You E, Yang JJ, Kim MJ, Cho SY, et al. Factors influencing discordant results of the QuantiFERON-TB Gold In-Tube test in patients with active TB. J Infect. 2013;67:28893.

33. Wang JY, Hsueh PR, Wang SK, Jan IS, Lee LN, Liaw YS, et al. Disseminated tuberculosis: a 10-year experience in a medical center. Medicine (Baltimore). 2007;86:39-46.

34. Yu SN, Jung J, Kim YK, Lee JY, Kim SM, Park SJ, et al. Diagnostic usefulness of IFN-gamma releasing assays compared with conventional tests in patients with disseminated tuberculosis. Medicine (Baltimore). 2015;94:e1094. 